\title{
Separation of uncertainty and variability in quantitative microbial risk assessment models
}

\author{
Maarten J. Nauta* \\ Microbiological Laboratory for Health Protection, National Institute of Public Health and the Environment (RIVM), P.O. Box 1, \\ 3720 BA Bilthoven, The Netherlands
}

Received 22 August 1999; received in revised form 1 December 1999; accepted 10 January 2000

\begin{abstract}
Quantitative risk assessment (QRA) modelling is increasingly used in food microbiology as a tool to evaluate health risks and to support the management of safe food production. Depending on the hazard and the process analysed, a QRA model may involve complex calculations: probability distributions are derived for the model parameters and the model is evaluated using specific risk analysis software. Second-order modelling, involving the separation of uncertainty and variability of model parameters, is considered of increasing importance in several fields of risk analysis. However, it is commonly neglected in microbial risk assessment studies. In this paper the relevance of second-order modelling in microbial risk assessment is illustrated by a simple example of a risk assessment of growth of B. cereus in pasteurised milk. It shows that the prediction of the outbreak size may depend on the way that uncertainty and variability are separated, and that a major outbreak may be overlooked if the distinction between uncertainty and variability is neglected. (C) 2000 Elsevier Science B.V. All rights reserved.
\end{abstract}

Keywords: Microbial risk assessment; Uncertainty; Variability; B. cereus; Milk

\section{Introduction}

Quantitative microbial risk analysis (QMRA) is increasingly being used as a tool to evaluate food production processes with regards to food safety and public health. Several integrated risk assessment studies have recently been completed or are in progress (e.g., on Salmonella enteritidis in eggs (Buchanan and Whiting, 1997; USDA 1998a), Es-

*Tel.: +31-30-2742-805; fax: +31-30-2744-434

E-mail address: maarten.nauta@rivm.nl (M.J. Nauta) cherichia coli O157:H7 in beef (Marks et al., 1998; Cassin et al., 1998a; USDA, 1999), Listeria monocytogenes in cheese (Bemrah et al., 1998). In these studies, the transmission of the hazard involved is modelled through a chain of processes, from animal production until consumption. The resulting model may be used to assess the current risk of the process and to predict the effects of different risk mitigation strategies.

A risk assessment can be performed at several levels of detail, depending on the specific food product and the attending hazard studied (Van Ger- 
wen and Zwietering, 1998; Van Gerwen et al., 2000). A rough, qualitative analysis may be sufficient to identify the potential hazards and the critical steps in the process, and to get an impression of the attendant risks. For a thorough quantitative risk assessment; however, the different steps in the production process must be described by specific models. Stochasticity may be incorporated into these models by using probability distributions for variable or uncertain model parameters. In that case, the model is usually analysed by means of Monte Carlo simulation. The output of the QMRA yields a risk estimate that should correctly reflect the uncertainty and variability in the model for the used data $(\mathrm{McNab}$, 1998; Cassin et al., 1998b).

Most QMRA studies published so far, are concentrating on the use of data to fit probability distributions of model parameters, that are to be used for the assessment (Bemrah et al., 1998; Cassin et al., 1998a), and the specific models that are to be used for the processing steps (Van Gerwen and Zwietering, 1998). These are challenging topics and important for a realistic risk assessment. Unfortunately, however, the specific interpretation of the resulting probability distributions gets little attention. When a Monte Carlo experiment is performed, samples are taken from the different distributions that are derived, without a careful consideration of the thing that the distributions stand for.

From this perspective the separation of uncertainty and variability as sources of variation of the model parameters is an important issue, intensely discussed in other fields of risk analysis (e.g., Hattis and Burmaster, 1994; Hoffman and Hammonds, 1994; Rai et al., 1996; Murphy, 1998; Anderson and Hattis, 1999). In this context, 'uncertainty' represents the lack of perfect knowledge of the parameter value, which may be reduced by further measurements. 'Variability', on the other hand, represents a true heterogeneity of the population that is a consequence of the physical system and irreducible by additional measurements (Murphy, 1998; Anderson and Hattis, 1999). At the moment, so-called second-order models (models that make the separation of the two) are scarcely produced in microbial risk assessment. Although uncertainty and variability are both mentioned as sources of variation, they are treated alike (e.g., Marks et al., 1998; McNab, 1998; Cassin et al., 1998a,b). This is caused by the fact that doing a
QMRA is laborious enough as it is, that it is often very difficult to separate uncertainty and variability on the basis of the available data, and that secondorder modelling requires more sophisticated simulation techniques.

In this paper we will illustrate why second-order models may be highly relevant for microbial risk assessment and we will show that separation of variability and uncertainty is not only necessary to estimate the uncertainty bounds on the risk estimate (Cassin et al., 1998a), but may also qualitatively affect the outcome of the risk assessment. In a straightforward example we will concentrate on microbial growth as a variable and uncertain process, that is a typical compound of many microbial risk assessments.

Clearly, the growth process is both variable (the growth curve of one bacterial population will never be exactly the same as that of another population, not even for the same strain under identical circumstances) and uncertain (we never know exactly how growth really progresses, because microbiological measurements used to construct a growth curve are always somehow imperfect). For the description of bacterial growth, a range of predictive food microbiology models can be used (see, e.g., McMeekin et al., 1993; Whiting, 1995; Van Gerwen and Zwietering, 1998). These models are highly valuable for the purpose of developing a safe food production process and HACCP, but have not been specifically developed for the purpose of QMRA. Most predictive models produce point estimates of growth only, and although some computer programs (like the Pathogen Modeling Program, USDA, 1998b) give a confidence interval around these estimates, such an interval most likely represents both uncertainty and variability and does not allow one to distinguish them.

The example below reflects a real risk assessment study by the fact that we have insufficient information on the source of variation of one of the parameters. We solve this problem by pretending that we are able to precisely separate between variability and uncertainty. By doing so, we illustrate that it may be better to improperly quantify their separation, than not to separate them at all. Our goal here is not to provide a general method for second-order QMRA modelling, but primarily to identify a potential problem in QMRA studies. The aim is to 
stimulate further discussion on second-order modelling in quantitative microbial risk assessment.

\section{Methods}

In the example below we construct a Monte Carlo simulation model for the growth of Bacillus cereus in pasteurised milk in a specific situation. To model the bacterial growth we use a simple predictive microbiology model that has been used by, e.g., Zwietering et al. (1996) and Notermans et al. (1998) for B. cereus in pasteurised milk.

As a primary growth model we assume exponential growth, neglecting the lag phase:

$N_{t}=N_{\mathrm{o}} \mathrm{e}^{k t}, \quad$ or $\ln \left(N_{t}\right)=\ln \left(N_{\mathrm{o}}\right)+k t$

where $N_{t}$ is the number of cells (cfu) at time $t, N_{\mathrm{o}}$ is the initial number of cells, and $k$ is the specific growth rate $\left(\mathrm{h}^{-1}\right)$. For a secondary growth model, temperature is incorporated by a square-root model (Ratkowsky et al., 1982).

$$
\sqrt{ } k=b\left(T-T_{\min }\right)
$$

where $k$ is the same parameter as in Eq. (1), $T$ is the growth temperature and $T_{\min }$ is the theoretical minimum growth temperature (both in ${ }^{\circ} \mathrm{C}$ ). The value of $b$ depends on additional growth conditions and the microorganism involved. For the parameters $b$ and $T_{\min }$ Zwietering et al. (1996) give $b=0.0354^{\circ} \mathrm{C}^{-1}$ $\mathrm{h}^{-0.5}$ and $T_{\min }=0^{\circ} \mathrm{C}$. As a simple secondary growth model Eq. (1) can therefore be rewritten as

$\ln \left(N_{t}\right)=\ln \left(N_{\mathrm{o}}\right)+b^{2} T^{2} t$

In our example we use this model equation, but in two different forms that are better suited for our objective. With $a=b^{2} \times 24 / \ln (10)=0.013^{\circ} \mathrm{C}^{-2}$ day $^{-1}$ Eq. (3) becomes

$$
\log \left(N_{t}\right)=\log \left(N_{\mathrm{o}}\right)+a T^{2} t
$$

and when we define one growth parameter $c=a T^{2} t$, this simplifies to

$$
\log \left(N_{t}\right)=\log \left(N_{\mathrm{o}}\right)+c
$$

For the Monte Carlo computer simulations @Risk for Windows 3.5.1 (Palisade, Newfield, USA) is used, running on an Excel 97 SR-2 (Microsoft) spreadsheet on a PC.

\section{Example}

Here we apply Eq. (5) to a QMRA problem, focussing on exposure assessment and bacterial growth. The objective of the example is to illustrate the difference in uncertainty and variability in a microbial risk assessment and to show why it is necessary to separate the two. It is not a complete QMRA. Some of the assumptions made may be disputed, but their aim is to keep the example simple and make it easier to focus on our objective.

The product of interest is pasteurised milk, and the hazard of concern is Bacillus cereus. For this pathogen no dose-response relationship is available. In this example a threshold dose of $10^{5}$ cfu per consumption dose is considered critical (Notermans et al., 1998).

\subsection{Problem}

Consider a vat with 1001 pasteurised milk stored between 0 and $4^{\circ} \mathrm{C}$. Ten samples of $10 \mathrm{ml}$ are taken from this vat and tested for the presence of $B$. cereus. It appears that one of the ten samples from the vat was tested positive with a test that has $100 \%$ sensitivity and $100 \%$ specificity. From this vat 100 cups containing 0.251 milk are taken and stored together at about $10^{\circ} \mathrm{C}$. After (exactly) 3 days of storage 100 people each drink one of the cups of milk.

The general problem is to quantitatively assess the consequences of this situation for public health.

\subsection{Solution}

To solve this hypothetical problem, we concentrate on three questions: (i) What are the numbers of B. cereus in the cups at the moment of consumption? (ii) What is the fraction of cups containing more than the critical level of $10^{5}$ cfu? (iii) What is the probability that more than 50 out of 100 cups contain more than $10^{5} \mathrm{cfu}$ in a cup?

Below, these questions are answered by three different methods. First, we make a 'deterministic estimate', without using probability distributions. 
Secondly, we derive probability distributions for the model parameters without separating uncertainty and variability. Thirdly, we do the same with making this distinction. The different results illustrate the relevance of using probability distributions for different model parameters, and the need to separate uncertainty and variability when applying these distributions.

\subsubsection{A deterministic estimate}

The growth parameter $c=a T^{2} t$ of Eq. (5) can be estimated as $c=0.013 \times 10 \times 10 \times 3=3.9$.

The initial number of cells in a cup, $N_{\mathrm{o}}$, has to be derived from the finding that one out of $1010-\mathrm{ml}$ samples from the vat is positive for B. cereus. For that purpose, consider the probability of no bacterial cells in a sample of $10 \mathrm{ml}$. According to the Poisson distribution this probability is $P(0)=\mathrm{e}^{-10 C}$, where $C$ $\left(\mathrm{ml}^{-1}\right)$ is the concentration in the vat. From the sample we estimate this probability as nine out of 10 , so $P(0)=0.9$. This gives $C=-\ln (0.9) / 10$ per $\mathrm{ml} \approx$ 0.01054 per $\mathrm{ml}$. So in $250 \mathrm{ml}$ we expect $250 \times$ $0.01054 \approx 2.63 \mathrm{cfu}$.

Therefore the point estimate of the final number of cells in a cup is $\log \left(N_{t}\right)=\log (2.63)+3.9 \approx 4.32$, so $N_{t} \approx 2.10^{4}$ cfu per cup.

This is a first version of an answer to question (i). This estimate is a little lower than the critical level, which implies no health risk of consumption of the milk. Questions (ii) and (iii) cannot be answered without the use of probability distributions.

\subsubsection{Using probability distributions without separation of uncertainty and variability}

To assess the probability of an exposure higher than the critical level, we have to implement the probability distributions of the model parameters. Below, we derive probability distributions for the parameters $N_{\mathrm{o}}$ and $c$ from model Eq. (5). In this section we do not separate between uncertainty and variability, as is a common practice in many currently published microbial risk assessment studies.

First, consider the initial number of cells in a cup, $N_{\mathrm{o}}$. Clearly, the value of $N_{\mathrm{o}}$ must be an integer and will be different in different cups. As the distribution of cells from the vat over the cups can be regarded as a Poisson process, the per cup variability can be given by a Poisson distribution. The mean of this distribution can be estimated as $\lambda=2.63$, as derived above. This mean, however, has a probability distribution too. It is derived from the concentration in the vat $C$, which is estimated from the finding that one from 10 samples is positive. This concentration is not known exactly: a range of values of $C$ could lead to the given sampling result.

The probability distribution of $C$ can be found by considering the sampling from the vat as a binomial process. ${ }^{1}$ Therefore, the uncertainty distribution of the probability $p$ of finding a negative sample can be described by a Beta distribution. Assuming that we have no information on $p$ before testing, we apply Bayes' theorem with a Uniform $(0,1)$ (or identically a $\operatorname{Beta}(1,1))$ prior. Now $p$ is given by $p=\operatorname{Beta}(n-s+$ $1, s+1)=\operatorname{Beta}(10,2)($ Vose, 1996, p. 58). Here, $n$ is the number of samples and $s$ is the number of positive samples. Combining this with $p=P(0)=$ $\mathrm{e}^{-10 C}$, as derived above for the deterministic estimate, gives a probability distribution of $C$ given by $-\ln (\operatorname{Beta}(10,2)) / 10$. The probability distribution of the mean value of $N_{\mathrm{o}}$ (the number of cells in $250 \mathrm{ml}$ ) is then given by $\lambda=-25 \ln (\operatorname{Beta}(10,2))$.

The distribution $B$. cereus cells over the cups remains a separate process. Without separating uncertainty and variability, the probability distribution of $N_{\mathrm{o}}$ per cup is a combination of both probability distributions:

$N_{\mathrm{o}} \sim$ Poisson $(-25 \ln (\operatorname{Beta}(10,2)))$.

Next, consider the growth parameter $c$. Its value may differ per cup and will not be known exactly, so it will also be described by a probability distribution. As $c=a T^{2} t$, this probability distribution can be assessed by considering the different parameters $a, T$ and $t$.

Parameter $a$ is related to the specific growth rate of B. cereus in milk (Zwietering et al., 1996). The probability distribution around its estimate $a=0.013$ is unknown. In an experimental study on the growth rates of different E. coli O157:H7 isolates (Nauta and Dufrenne, 1999) it has been found that this growth rate has a standard deviation of a little less than one-tenth of its mean value. We use this finding to assume here that parameter $a$ has a Normal $N(0.013,0.001)$ distribution.

\footnotetext{
${ }^{1}$ This method has been adapted from a course in quantitative risk analysis from David Vose Risk Analysis Consultancy in 1998.
} 
The storage temperature $T$ will also have a probability distribution, because it will vary and will not be precisely known, depending for example on the refrigerator where the milk has been stored and the quality of the thermometer in it. Here we assume some imaginary 'expert opinion' on this, which states that $T$ has a Normal $N(10,1)$ distribution.

For simplicity we assume that the storage time $t$ is exactly 3 days.

The resulting probability distribution of $c=a T^{2} t$, shown in Fig. 1, is constructed by a Latin Hypercube (Monte Carlo) experiment of 10000 samples from the distributions of $a$ and $T$. Note that this distribution is not symmetrical.

The pathogen numbers in the 100 cups in the example are simulated by calculating $\log \left(N_{t}\right)$ with Eq. (4) using independent Monte Carlo samples from the distributions of $N_{\mathrm{o}}, T$ and $a$ from the distributions derived above. The resulting distributions of $\log \left(N_{t}\right)$ for 10 series of 100 cups are given in Fig. 2a. In a series of 1000 runs with 100 cups the mean number of cups with $\log \left(N_{t}\right)>5$ was 26.7 , with standard deviation 4.51. In none of the 1000 runs 50 or more cups (from 100) were contaminated above the critical level.

\subsubsection{Second-order modelling: separation of uncertainty and variability}

We now focus on the separation of uncertainty and variability.

\section{Distribution for $c=a T^{2} t$}

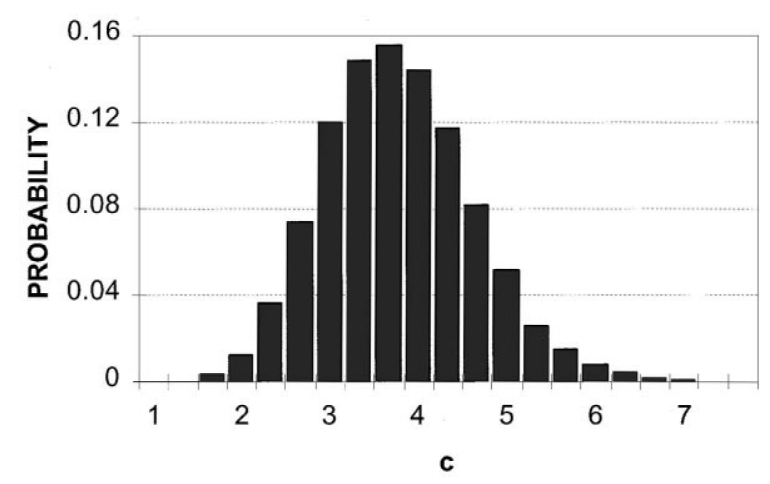

Fig. 1. The probability distribution of the growth parameter $c=$ $a T^{2} t$ from Eq. (5), derived from a Normal $N(10,1)$ distribution of the growth temperature $T$ and a Normal $N(0.013,0.001)$ distribution of the parameter $a$, with $t=3$ days.
First consider the probability distribution of $N_{\mathrm{o}}$, as derived in the previous section.

The number of cells in the vat is uncertain. It has one, unknown, value, which is estimated using the (for this purpose) rather imprecise instrument of qualitatively studying 10 samples of $10 \mathrm{ml}$. The mean value of $N_{\mathrm{o}}$ in a cup, $\lambda$, is linearly related to the number of cells in the vat. This number is unknown, estimated from the finding of one positive sample from 10. Therefore the distribution of the mean, $\lambda=-25 \ln (\operatorname{Beta}(10,2))$ as derived above, is an uncertainty distribution.

Given this mean number, the initial number of cells in each of the 100 cups, $N_{\mathrm{o}}$, differs per cup and is variable. So the Poisson $(\lambda)$ distribution describing the probability of $N_{\mathrm{o}}$ per cup, is a variability distribution.

Next, consider the probability distribution of $c$.

If this distribution represents uncertainty only, it means that the increase is identical (but unknown) in each of the 100 cups. If, on the other hand, this distribution represents variability only, this means that we are confident that the distributions do only represent the per cup variability. This implies for instance that the probability distribution of the temperature is a per cup variability. As neither of these extremes seems realistic, we have to construct some 'in between' situation that accommodates both uncertainty and variability. For this purpose we introduce the parameter $\alpha_{x}$ representing the fraction of the total variance attributable to uncertainty for parameter $x$ (such that $1-\alpha_{x}$ represents the fraction of the total variance attributable to variability). If the final probability distribution of parameter $x$ is $\operatorname{Normal}(\mu, \sigma)$, the uncertainty distribution is defined as $N\left(\mu, \sigma \vee \alpha_{x}\right)$. For $x_{\mathrm{u}}$, a sample of this uncertainty distribution, the variability distribution is $N\left(x_{\mathrm{u}}, \sigma \vee\left(1-\alpha_{x}\right)\right)$. (Note that if $\alpha_{x}=1, N(\mu, \sigma)$ represents an uncertainty distribution and if $\alpha_{x}=0$, $N(\mu, \sigma)$ represents a variability distribution.)

We evaluate $c$ for three situations with different values for the 'uncertainty attributable fraction' of the temperature, $\alpha_{T}$, and the 'uncertainty attributable fraction' for parameter $a, \alpha_{a}$ : (a) the probability distributions of $T$ and $a$ represent variability only, so $\alpha_{T}=\alpha_{a}=0 \quad$ (' $\alpha=0$ '); (b) half the variance in the probability distributions of both $T$ and $a$ represents uncertainty, and the other half represents variability, so $\alpha_{T}=\alpha_{a}=0.5$ (' $\alpha=0.5$ '); (c) the probability dis- 
no separation

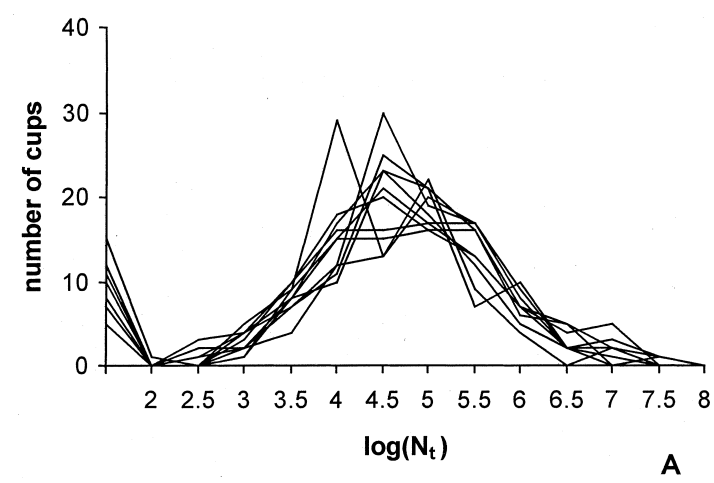

$\alpha=0.5$

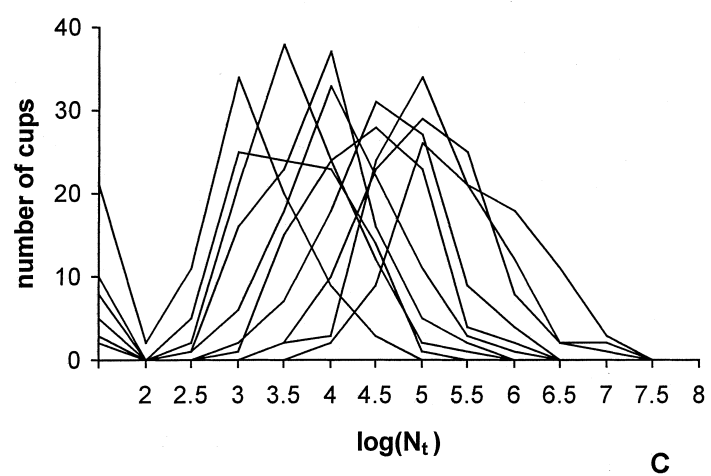

$\alpha=0$

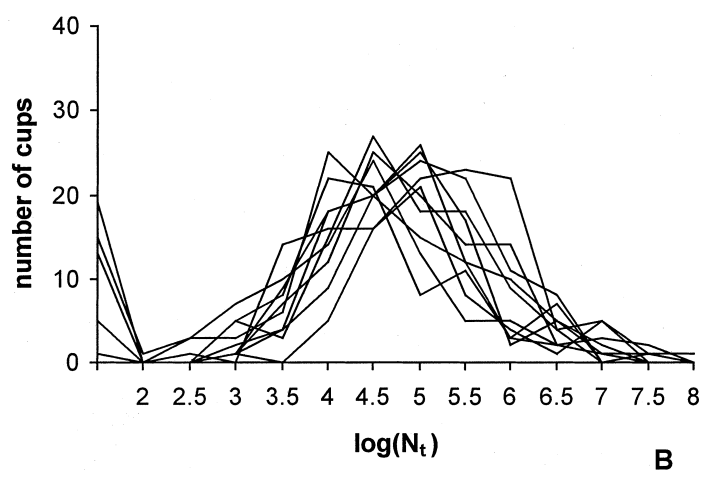

$\alpha=1$

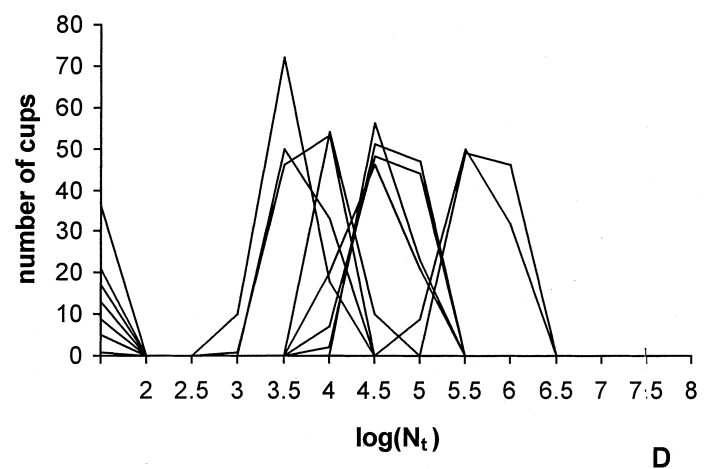

Fig. 2. The results of 10 runs of a Monte Carlo simulation on 100 cups of milk. The distribution $\operatorname{of} \log \left(N_{t}\right.$,), the number of cells in the cups, is given for each run. The indicated numbers on the $x$-axis are upper limits of classes. The numbers of cups without $B$. cereus $\left(N_{t}=0\right)$ are given at the intersection with the $y$-axis. Individual lines show the variability between cups, whereas differences between lines indicate the uncertainty. (a) The results when uncertainty and variability are not separated. The differences between the lines are solely a consequence of random sampling. In (b)-(d) variability and uncertainty are separated: (b) $\alpha=0$, so only the number of $B$. cereus in the vat is uncertain; (c) $\alpha=0.5$, so the increase, as expressed by the parameter $c$, is both variable and uncertain. For (d) $\alpha=1$, so the increase is uncertain, not variable. Here, only the initial number of cells in the cups is variable. Note that the difference between total number of cups with log $\left(N_{t}\right)>5$ in the different runs is smallest in (a) and largest in (d), as shown in more detail in Fig. 3.

tributions of $T$ and $a$ represent uncertainty only, so $\alpha_{T}=\alpha_{a}=1$ (' $\alpha=1$ ').

The separation of uncertainty and variability is implemented in Monte Carlo simulations by first sampling once from the uncertainty distributions of $N_{\mathrm{o}}, a$ and $T$ for a set of 100 cups, and then sampling from the variability distributions of these parameters for each of the 100 cups.

The results for $\log \left(N_{t}\right)$ for 10 runs of 100 cups in all three cases are shown in Fig. 2b-d.

The uncertainty distribution of the number of cups containing more than the critical level of $\log \left(N_{t}\right)=5$ (question (ii)) is studied by running 1000 Monte
Carlo simulations for 100 cups, resulting in (mean \pm standard deviation): (a) $25.9 \pm 12.0$, (b) $25.6 \pm 24.7$, (c) $26.6 \pm 39.1$ cups. Note that the mean numbers are almost the same in all simulations, but that the standard deviation increases significantly. As the standard deviation is a measure of uncertainty here, it is not surprising that the standard deviation increases with the value of $\alpha$.

The percentages of simulations where 50 or more (from 100) of the cups were contaminated above the critical level (question (iii)) are 3.4, 18.3 and 27.4\%, respectively. Apparently, the probability of a large number of heavily contaminated cups increases when 
the uncertainty increases at the expense of the variability. This is illustrated in Fig. 3, which depicts the results of the 1000 Monte Carlo runs as uncertainty distributions. With the $10^{5}$ threshold doseresponse model assumed here, the number of cups containing more than $\log \left(N_{t}\right)=5$ is equivalent to the outbreak size. The figure shows the (estimated) probabilities of an outbreak size larger than or equal to the number given on the horizontal axis, for different assumptions on the nature of the input distributions. It compares the uncertainty about the outbreak size of the three cases studied with separation of uncertainty and variability $(\alpha=0, \alpha=0.5$ and $\alpha=1$ ) and two extreme cases: (1) without separation of variability and uncertainty, where it is implicitly assumed that all probability distributions used are variability distributions; and (2) the result of the imaginary case where all distributions are assumed to represent uncertainty distributions.

\section{Discussion}

In the example given above we illustrated that the interpretation of (the probability distribution of) the input of a quantitative microbial risk assessment (QMRA) study may be decisive for the qualitative outcome of the risk assessment. Using a deterministic estimate only, the analysis concluded that there was no risk. Using a stochastic model without

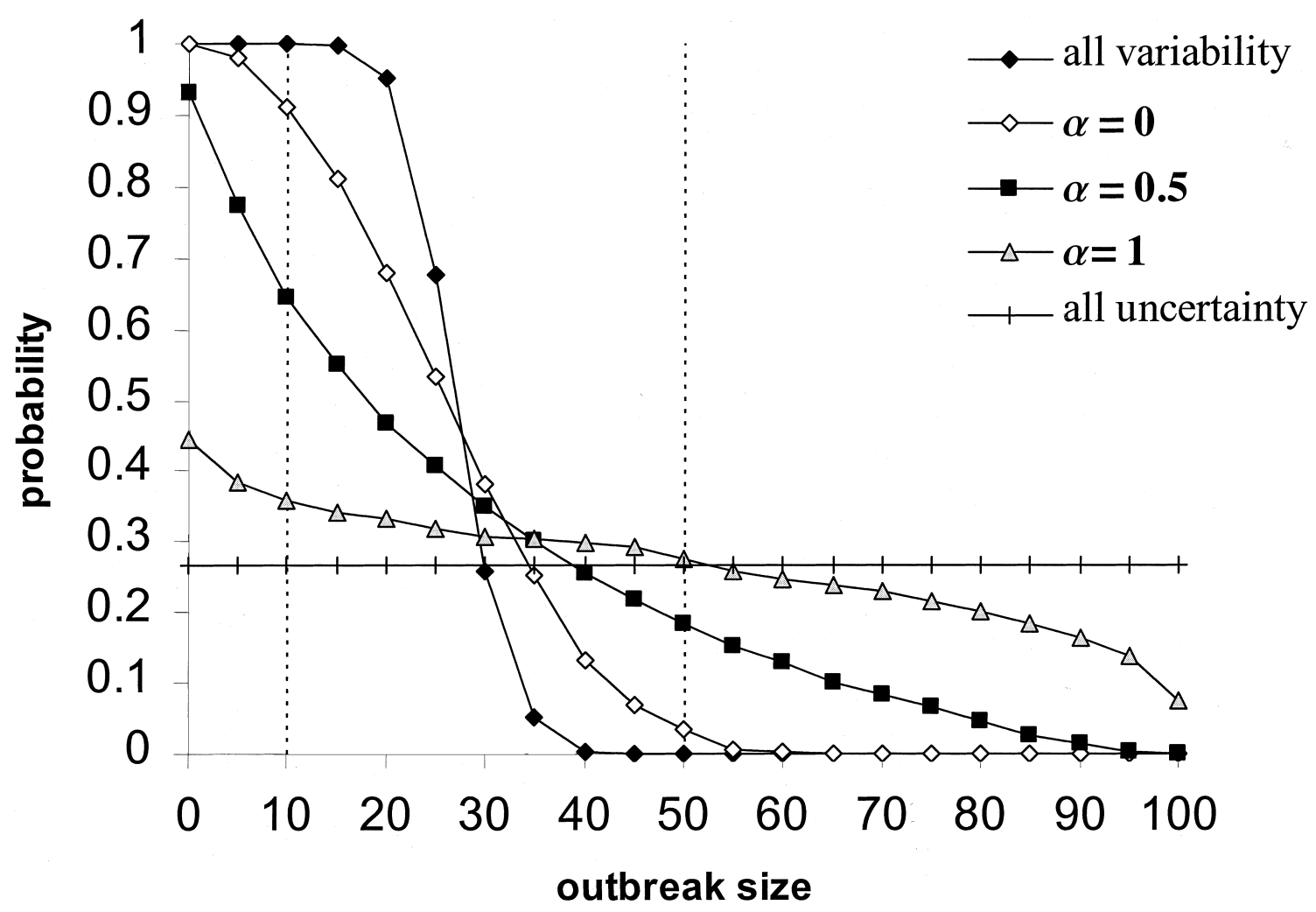

Fig. 3. The uncertainty distributions of the outbreak size as found in four sets of 1000 simulation runs with 100 cups, and in the extreme case that all input distributions represent uncertainty. The outbreak size is equivalent to the number of cups containing more than the threshold number of $10^{5} \mathrm{cfu}$ per cup. The probabilities on the vertical axis are the estimated probabilities of an outbreak size larger than or equal to the number given on the horizontal axis. The dashed lines point at the probabilities of an outbreak size of at least 10 or 50 people. Without separation of variability and uncertainty, all input distributions are assumed to be represent variability, and the predicted outbreak size is always larger than 10 and smaller than 50. When variability and uncertainty are separated (with $\alpha=0, \alpha=0.5$ and $\alpha=1$ ), the probability of an outbreak size of at least 10 decreases, but the probability of an outbreak of size 50 increases. If all input distributions would represent uncertainty, the outbreak size is either 0 or 100 . 
separation of uncertainty and variability, we identified an individual risk, but no major outbreak with more than 50 people involved. And with a secondorder risk assessment model, where uncertainty and variability are separated, the outcome was that there is a potential individual risk and a potential major outbreak.

These qualitative conclusions may be disputed as they depend on for instance the definitions of an outbreak and the critical number of cells per consumption dose. In practice, a deterministic estimate of the contamination level that is a little below the critical level will not lead to the conclusion that the product is safe. In that respect the conclusions of the deterministic and stochastic approaches will be the same. But whereas a decision maker will apply some rough 'safety margin' around a deterministic estimate to express his feeling of uncertainty, the specific aim of QMRA is to quantify this uncertainty about the safety of the process. The example shows that this quantification of uncertainty in a QMRA may lead to a false conclusion when uncertainty and variability are not separated.

As the example has been constructed for the purpose of illustrating the relevance of second-order modelling, it deliberately does not describe a complete QMRA. Adding models that make the example more realistic, would also make it more complex and does not contribute to its transparency. Even for the very short 'food production process' described, several simplifying assumptions were made. Among these are a simple exponential growth model without a lag phase, a constant and known minimum temperature of growth, a perfect test for B. cereus used for sampling from the vat and a one-value threshold dose-response model. It should be realised that the effect of incorporating a more precise description of these aspects on the final risk estimate may possibly exceed the illustrated effect of (not) separating uncertainty and variability in growth. However, extending the model by incorporating additional parameters and models, will also give an increase in sources of variability and uncertainty. To evaluate their effect, second-order modelling should be considered throughout the model. If second-order modelling is important in a simple model, there is no reason to assume that it will be cancelled out in a complex model.

Being a specifically designed QMRA problem, the example does not prove that separation of uncertainty and variability is crucial in all situations. The specific purpose of the QMRA, that is the exact research question to be answered, is very important here. When, in the example, the focus would have been on one person drinking one cup only, things would have been different. In that case the variability between cups would not have been relevant, as there was no 'population of cups' defined. In this context, note that the mean probability that ' $a$ ' cup is contaminated with more than the critical level is about $\hat{p}=0.26$ in all models analysed, both with and without separation of uncertainty and variability.

The probability distribution of this 'probability that a cup is contaminated above the critical level', $p$, should be interpreted as an uncertainty distribution. If uncertainty and variability are not separated, and it is implicitly assumed that all input distributions are variability distributions, $p$ is a single fixed value without an uncertainty distribution. In that case sampling from 100 cups is a binomial process with sample size $n=100$ and probability $p$. With $p=\hat{p}=$ $26.7 / 100=0.267$, this distribution predicts a standard deviation of 4.42 , almost the same as the value 4.51 found in our simulations. A plot of this $\operatorname{Binomial}(100,0.267)$ overlaps with the 'all variability' curve in Fig. 3.

If we have uncertainty in the input distributions, $p$ is uncertain too. As illustrated in Fig. 3. this results in a wider spread of the number of cups contaminated above the critical level and thus in an increasing uncertainty about the outbreak size. As a result the uncertainty about the outbreak size increases with increasing uncertainty in the input distributions. The probability of an outbreak of less than about 25 people is larger when the uncertainty about the input is small, but the probability of a larger outbreak increases with increasing uncertainty. In the extreme and unrealistic case that all input probability distributions would reflect uncertainty, there would be either no outbreak (with probability $1-p$ ) or an outbreak with all 100 people (with probability $p$ ).

The separation of uncertainty and variability is sometimes straightforward, as in the example for the initial number in a cup $N_{\mathrm{o}}$. On the other hand it can be very difficult to properly separate the two, as with the growth parameter $c$. Unfortunately the latter is generally the case. Often one has very little idea about even the form of the distribution, let alone 
about its underlying uncertainty and variability. There is however no reason to assume that the easiest solution, treating uncertainty or variability as one, is also the best solution to this problem. In that case the distributions are implicitly assumed to represent either variability or uncertainty only. When the nature of the probability distribution of a parameter is unclear and holds both uncertainty and variability, the best solution may be to assume something half way, like we did with the option $\alpha=0.5$. In that way the implicit assumptions about uncertainty and variability can be made explicit. By making explicit assumptions, the effect of these assumptions can be explored, and the relevance of second-order modelling may become manifest. Also, with some extra information the uncertainty may be reduced. For the situation described in the example, additional experiments may help. If the example is simulated by an experiment with 100 cups, with measurements of the concentrations in the cups, the distribution found may be highly informative.

The conclusion of this study is that it is important to be aware of the nature of the probability distributions used in a QMRA. For every probability distribution applied in the risk assessment, both input and output, the risk assessor has to realise what these distributions stand for. Second-order modelling may be necessary to arrive at a proper risk estimate. When uncertainty and variability are not separated, it is implicitly assumed that either one or the other is negligible. Our example illustrates the potential impact of this implicit assumption on the risk estimate. For a transparent risk assessment the assumptions on uncertainty and variability should therefore be made explicit. When the nature of the probability distribution of a model parameter is not clear, it may be better to imprecisely assign a parameter as uncertain and/or variable than not to separate them at all.

\section{Acknowledgements}

This research was carried out under project number 257851 on behalf of the directory board of the National Institute of Public Health and the Environment (RIVM). The author thanks P.F.M. Teunis and A.H. Havelaar for helpful comments on earlier versions of the manuscript.

\section{References}

Anderson, E.L., Hattis, D., 1999. Uncertainty and variability. Risk Anal. 19, 47-49.

Bemrah, N., Sanaa, M., Cassin, M.H., Griffiths, M.W., Cerf, O., 1998. Quantitative risk assessment of human listeriosis from consumption of soft cheese made from raw milk. Prev. Vet. Med. 37, 129-145.

Cassin, M.H., Lammerding, A.M., Todd, E.C.D., Ross, W., McColl, R.S., 1998a. Quantitative risk assessment for Escherichia coli $\mathrm{O} 157: \mathrm{H} 7$ in ground beef hamburgers. Int. J. Food Microbiol. 41, 21-44.

Cassin, M.H., Paoli, G.M., Lammerding, A.M., 1998b. Simulation modeling for microbial risk assessment. J. Food Prot. 61, $1560-1566$.

Hattis, D., Burmaster, D.E., 1994. Assessment of variability and uncertainty distributions for practical risk analyses. Risk Anal. 14, 713-730.

Hoffman, F.O., Hammonds, J.S., 1994. Propagation of uncertainty in risk assessments: the need to distinguish between uncertainty due to lack of knowledge and uncertainty due to variability. Risk Anal. 14, 707-712.

Marks, H.M., Coleman, M.E., Lin, C.-T.J., Roberts, T., 1998. Topics in microbial risk assessment: dynamic flow tree processes. Risk Anal. 18, 309-328.

McMeekin, T.A., Olley, J.N., Ross, T., Ratkowsky, D.A., 1993. In: Predictive Microbiology: Theory and Application, Wiley, New York, p. 340.

McNab, W.B., 1998. A general framework illustrating an approach to quantitative microbial food safety risk assessment. J. Food Prot. 61, 1216-1228.

Murphy, B.L., 1998. Dealing with uncertainty in risk assessment. Human Ecol. Risk Assess. 4, 685-699.

Nauta, M.J., Dufrenne, J., 1999. Variability in growth characteristics of different E. coli $\mathrm{O} 157: \mathrm{H} 7$ isolates, and its implications for predictive microbiology. Quant. Microbiol. (in press).

Notermans, S., Nauta, M.J., Jansen, J., Jouve, J.L., Mead, G.C., 1998. A risk assessment approach to evaluating food safety based on product surveillance. Food Control. 9, 217-223.

Rai, S.N., Krewski, D., Bartlett, S., 1996. A general framework for the analysis of uncertainty and variability in risk assessment. Human Ecol. Risk Assess. 2, 972-989.

Ratkowsky, D.A., Olley, J., McMeekin, T.A., Ball, A., 1982. Relationship between temperature and growth rate of bacterial cultures. J. Bacteriol. 149, 1-5.

USDA, 1998a. Salmonella enteritidis Risk Assessment. http:/ /www.fsis.usda.gov/ophs/risk/index.htm

USDA, 1998b. Pathogen Modeling Program for Windows, version 5.1. Microbial Food Safety Research Unit, USDA, ARS Eastern Regional Research Centre, http://www.arserrc.gov/ mfs/pathogen.htm, Philadelphia.

USDA, 1999. Escherichia coli O157:H7 in beef risk assessment project. http://www.fsis.usda.gov/ophs/ecolrisk/home.htm.

Van Gerwen, S.J.C., Zwietering, M.H., 1998. Growth and inactivation models to be used in quantitative risk assessments. J. Food Prot. 61, 1541-1549.

Van Gerwen, S.J.C., Te Giffel, M.C., Van't Riet, K., Zwietering, M.H., 2000. Stepwise quantitative risk assessment as a tool for characterisation of microbial food safety. (Submitted). 
Vose, D., 1996. In: Quantitative Risk Analysis: A Guide To Monte Carlo Simulation Modelling, Wiley, Chichester, p. 328.

Whiting, R.C., 1995. Microbial modeling in foods. Crit. Rev. Food Sci. Nutr. 35, 467-494.

Whiting, R.C., Buchanan, R.L., 1997. Development of a quantitative risk assessment model for Salmonella enteritidis in pasteurized liquid eggs. Int. J. Food Microbiol. 36, 111-125.
Zwietering, M.H., De Wit, J.C., Notermans, S., 1996. Application of predictive microbiology to estimate the number of Bacillus cereus in pasteurised milk at the point of consumption. Int. J. Food Microbiol. 30, 55-70. 